\title{
Backdoors for Linear Temporal Logic
}

\author{
Arne Meier ${ }^{1}$. Sebastian Ordyniak ${ }^{2}$ (D) M. S. Ramanujan ${ }^{3}$. Irena Schindler ${ }^{1}$
}

Received: 31 May 2017 / Accepted: 5 September 2018 / Published online: 18 September 2018

(c) The Author(s) 2018

\begin{abstract}
In the present paper, we introduce the backdoor set approach into the field of temporal logic for the global fragment of linear temporal logic. We study the parameterized complexity of the satisfiability problem parameterized by the size of the backdoor. We distinguish between backdoor detection and evaluation of backdoors into the fragments of Horn and Krom formulas. Here we classify the operator fragments of globallyoperators for past/future/always, and the combination of them. Detection is shown to be fixed-parameter tractable whereas the complexity of evaluation behaves differently. We show that for Krom formulas the problem is paraNP-complete. For Horn formulas, the complexity is shown to be either fixed parameter tractable or paraNP-complete depending on the considered operator fragment.
\end{abstract}

Keywords Linear temporal logic $\cdot$ Parameterized complexity $\cdot$ Backdoor sets

A preliminary version of this work was presented at IPEC 2016 [28].

Sebastian Ordyniak

sordyniak@gmail.com

Arne Meier

meier@thi.uni-hannover.de

M. S. Ramanujan

ramanujan@ac.tuwien.ac.at

Irena Schindler

schindler@thi.uni-hannover.de

1 Institut für Theoretische Informatik, Leibniz Universität Hannover, Appelstrasse 4, 30167 Hannover, Germany

2 Algorithms Group, University of Sheffield, Regent Court, 211 Portobello, Sheffield S1 4DP, UK

3 Institut für Computergraphik und Algorithmen 186/1, Technische Universität Wien, Favoritenstraße 9-11, 1040 Wien, Austria 


\section{Introduction}

Temporal logic is one of the most important formalism in the area of program verification and validation of specification consistency. Most notable are the seminal contributions of Kripke [21], Pnueli [32], Emerson, Clarke, and Halpern [7,14] to name a few. There exist several different variants of temporal logic from which, best known are the computation tree logic CTL, the linear temporal logic LTL, and the full branching time logic CTL* . In this paper, we will consider the global fragment of LTL for formulas in separated normal form (SNF) which has been introduced by Fisher [15]. This normal form is a generalization of the conjunctive normal form from propositional logic to linear temporal logic with future and past modalities interpreted over the flow of time, i.e., the frame of the integers $(\mathbb{Z},<)$. In SNF the formulas are divided into a past, a present, and a future part. Technically this normal form is not a restriction since one can always translate an arbitrary LTL formula to a satisfiability-equivalent formula in SNF in time linear in the original formula [15]. In fact, the restriction to SNF normal form is crucial for us, because it is known that syntactical restrictions of arbitrary LTL formulas such as Horn or Krom do not lead to tractability [4].

LTL and its two main associated computational problems LTL model checking and LTL satisfiability have been deeply investigated in the past. In this work we focus on the LTL satisfiability problem, i.e., given an LTL formula the question is whether there is a temporal interpretation that satisfies the formula. Sistla and Clarke classified the computational complexity of the satisfiability problem to be PSPACE-complete [36]. Then, later, several restrictions of the unrestricted problem have been considered. These approaches considered operator fragments [29], Horn formulas [4], temporal operator fragments, temporal depth, and number of propositional variables [8], the use of negation [27], an XOR fragment [11], an application of Post's lattice [3], and the SNF fragment [2].

In contrast to LTL satisfiability where the search for fruitful parameterization has so far been rather unsuccessful [26], various important parameterizations have been identified for the satisfiability problem of propositional formulas (SAT) [5,30,37]. One very prominent and well-studied structural parameterization for SAT are so-called backdoor sets. Informally, backdoors are small sets of variables of a SAT instance that represent "clever reasoning shortcuts" through the search space. Backdoor sets have been widely used in the areas of propositional satisfiability $[9,10,19,20,33,35,38]$, and also for material discovery [25], abductive reasoning [31], argumentation [13], planning [22,23], and quantified Boolean formulas [34]. A backdoor set is defined with respect to some fixed base class for which the computational problem under consideration is polynomial-time tractable. For instance, in the case of SAT, a backdoor set $B$ for a given CNF formula $\phi$ into the base class of Horn formulas is a set of variables such that for every assignment of the variables in $B$ it holds that the reduced formula, i.e., the formula obtained after applying the assignment to $\phi$, is Horn. Given such a backdoor set one can decide the satisfiability of $\phi$ in time $O\left(2^{|B|} p(|\phi|)\right)$ by enumerating the $2^{|B|}$ assignments of the variables in $B$ and for each such assignment solving the remaining formula in time $p(|\phi|)$, where $p$ is a polynomial given by the base class. As a result, once a small backdoor set is identified the satisfiability check is fixed-parameter tractable for the parameter backdoor size. Since the backdoor set 
Table 1 Results overview

\begin{tabular}{|c|c|c|c|c|c|}
\hline Problem & Operators & HORN & & KROM & \\
\hline Detection & Any & FPT & (Thm. 5) & FPT & (Thm. 5) \\
\hline \multirow[t]{3}{*}{ Evaluation } & $\circledast$ & FPT & (Thm. 8) & paraNP-c. & (Thm. 9) \\
\hline & $\square_{\mathrm{F}}, \square \mathrm{P}$ & paraNP-c. & (Thm. 10) & paraNP-c. & (Above) \\
\hline & One of $\square_{\mathrm{F}}, \square_{\mathrm{P}}$ & Open & & paraNP-c. & (Cor. 11) \\
\hline \multirow[t]{2}{*}{ LTL-SAT } & *⿻ $, \square_{\mathrm{F}}, \square_{\mathrm{P}}$ & $P[2]$ & & NP-c. [2] & \\
\hline & 头 & $P[2]$ & & NL [2] & \\
\hline
\end{tabular}

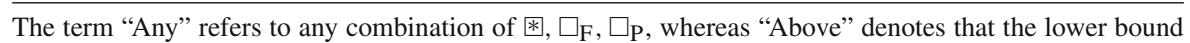
from the cell above applies

is usually not provided with the input, it is crucial that small backdoor sets to a given base class can be found efficiently. When employing the backdoor approach one consequently usually considers two subtasks the so-called detection and evaluation problem, where the former is the task to identify a small backdoor set and the later concerns the solution of the problem using the backdoor set.

Our Contribution In this paper, we introduce a notion of backdoors for the global fragment of LTL formulas that are given in SNF. Namely, we consider backdoor sets to the base classes that have recently been identified by Artale et al. [2]. These base classes are defined by both restrictions on the allowed temporal operators (i.e., to a subset of $\left\{\right.$ 困, $\square_{\mathrm{P}}, \square_{\mathrm{F}}$ \}) and restrictions on the clauses to be either HORN or KROM. We show that surprisingly a notion of backdoor sets very similar to the strong backdoor sets employed for SAT [18] can also be successfully applied to LTL formulas. Whereas the detection of these backdoor sets can be achieved via efficient fpt-algorithms for all the considered fragments (using algorithms similar to the algorithms employed in the context of SAT), the evaluation of these backdoor sets turns out to be much more involved. In particular, we obtain tractability of the evaluation problem for HORN formulas using only the always operator. In fact, LTL restricted to only the always operator, is already quite interesting, since it allows one to express "Safety" properties of a system. For almost all of the remaining cases we show that the evaluation problem is paraNPhard. Moreover, the techniques used to show these results are very different from and more involved than the techniques employed for SAT, i.e., in the context of SAT the backdoor set evaluation problem is trivial. Our results are summarized in Table 1.

\section{Preliminaries}

Parameterized Complexity A good introduction into the field of parameterized complexity is given by Downey and Fellows [12]. A parameterized problem $\Pi$ is a tuple $(Q, \kappa)$ such that the following holds. $Q \subseteq \Sigma^{*}$ is a language over an alphabet $\Sigma$, and $\kappa: \Sigma^{*} \rightarrow \mathbb{N}$ is a computable function; then $\kappa$ also is called the parameterization (of $\Pi$ ).

If there is a deterministic Turing machine $M$ and a computable function $f: \mathbb{N} \rightarrow \mathbb{N}$ s.t. for every instance $x \in \Sigma^{*}$ (i) $M$ decides correctly if $x \in Q$, and (ii) $M$ has a runtime 
bounded by $f(\kappa(x)) \cdot|x|^{O(1)}$, then we say that $M$ is an fpt-algorithm for $\Pi$ and that $\Pi$ is fixed-parameter tractable (or in the class FPT). If $M$ is non-deterministic, then $\Pi$ belongs to the class paraNP. One way to show paraNP-hardness of a parameterized problem $(Q, \kappa)$ is to show that $Q$ is NP-hard for a specific, fixed value of $\kappa$, i.e., there exists a constant $\ell \in \mathbb{N}$ such that $(Q, \kappa)_{\ell}:=\{x \mid x \in Q$ and $\kappa(x)=\ell\}$ is NP-hard.

Temporal Logic We assume familiarity with standard notions of propositional logic. Let PROP be a finite set of propositions and $\perp / T$ abbreviate the constants false/true. The syntax of the global fragment of LTL is defined by the following EBNF:

$$
\varphi::=\perp|\top| p|\neg \varphi| \varphi \wedge \varphi|\varphi \vee \varphi| \square_{\mathrm{P}} \varphi\left|\square_{\mathrm{F}} \varphi\right| \circledast \varphi,
$$

where $p \in$ PROP. Here $\square \mathrm{P} \varphi$ can be read as " $\varphi$ holds in every point in the past", $\square_{\mathrm{F}} \varphi$ as " $\varphi$ holds in every point in the future", and $* \varphi$ as " $\varphi$ holds always". We also will make use of well-known shortcuts such as $\rightarrow, \leftrightarrow$. Now we define the semantics of these formulas. Here, we interpret LTL formulas over the flow of time $(\mathbb{Z},<$ ) (for further information on this approach, see, e.g., Gabbay et al. [17]). Note that all our results can be easily transferred to the case if the formulas are evaluated over the set of natural numbers instead of the set of all integers.

Definition 1 (Temporal Semantics) Let PROP be a finite set of propositions. A temporal interpretation $\mathfrak{M}=(\mathbb{Z},<, V)$ is a mapping from propositions to moments of time, i.e., $V:$ PROP $\rightarrow \mathcal{P}(\mathbb{Z})$. The satisfaction relation $\models$ is then defined as follows where $n \in \mathbb{Z}, \varphi, \psi \in \mathrm{LTL}$

\begin{tabular}{|c|c|c|}
\hline $\mathfrak{M}, n \models \top$ & & always, \\
\hline $\mathfrak{M}, n \models \perp$ & & never, \\
\hline $\mathfrak{M}, n \models p$ & iff & $n \in V(p)$ \\
\hline $\mathfrak{M}, n \models \neg \varphi$ & iff & $\mathfrak{M}, n \not \models \varphi$, \\
\hline $\mathfrak{M}, n \models \varphi \vee \psi$ & iff & $\mathfrak{M}, n=\varphi$ or $\mathfrak{M}, n \models \psi$, \\
\hline $\mathfrak{M}, n \models \varphi \wedge \psi$ & iff & $\mathfrak{M}, n \models \varphi$ and $\mathfrak{M}, n \models \psi$, \\
\hline $\mathfrak{M}, n \models \square_{\mathrm{F}} \varphi$ & iff & for all $k>n$ it holds $\mathfrak{M}, k \models \varphi$, \\
\hline $\mathfrak{M}, n \models \square \mathrm{P} \varphi$ & iff & for all $k<n$ it holds $\mathfrak{M}, k \models \varphi$, and \\
\hline $\mathfrak{M}, n \models \circledast \varphi$ & iff & for all $k \in \mathbb{Z}$ it holds $\mathfrak{M}, k \models \varphi$. \\
\hline
\end{tabular}

We say that $\varphi$ is satisfiable if there is a temporal interpretation $\mathfrak{M}$ such that $\mathfrak{M}, 0 \models$ $\varphi$. Then $\mathfrak{M}$ is also referred to as a (temporal) model (of $\varphi$ ). Sometimes we also directly write $\mathfrak{M}(p)$ instead of $V(p)$.

Table 2 exemplifies the semantics with some basic formulas. As shown by Fisher et al. every LTL formula considered over the frame $(\mathbb{Z},<)$ has a satisfiabilityequivalent formula in the separated normal form SNF [16], which can be constructed in linear time [15]). We follow the notation of SNF formulas by Artale et al. [2] and directly restrict them to the relevant global fragment of this study:

$$
\begin{gathered}
\lambda::=\perp|p| \square_{\mathrm{F}} \lambda\left|\square_{\mathrm{P}} \lambda\right| \circledast \lambda, \\
\varphi::=\lambda|\neg \lambda| \varphi \wedge \varphi \mid \circledast(\neg \lambda \vee \cdots \vee \neg \lambda \vee \lambda \vee \cdots \lambda),
\end{gathered}
$$

where $\lambda$ is called a temporal literal and $\varphi$ is said to be in clausal normal form. 
Table 2 Temporal semantics

\begin{tabular}{|c|c|c|c|c|c|c|c|c|}
\hline & $<0$ & 0 & 1 & 2 & 3 & 4 & 5 & $>5$ \\
\hline$p$ & 1 & 1 & 0 & 1 & 0 & 1 & 1 & 0 \\
\hline$q$ & 0 & 0 & 1 & 1 & 0 & 0 & 1 & 1 \\
\hline$r$ & 0 & 0 & 0 & 0 & 1 & 0 & 0 & 0 \\
\hline$p \wedge q$ & 0 & 0 & 0 & 1 & 0 & 0 & 1 & 0 \\
\hline 圈 $p$ & 0 & 0 & 0 & 0 & 0 & 0 & 0 & 0 \\
\hline$\square_{\mathrm{F}} q$ & 0 & 0 & 0 & 0 & 0 & 1 & 1 & 1 \\
\hline$\square_{\mathrm{F}} \square_{\mathrm{F}} q$ & 0 & 0 & 0 & 0 & 1 & 1 & 1 & 1 \\
\hline$\square \mathrm{P} p$ & 1 & 1 & 1 & 0 & 0 & 0 & 0 & 0 \\
\hline 困( $\square \mathrm{p} p)$ & 0 & 0 & 0 & 0 & 0 & 0 & 0 & 0 \\
\hline$\circledast\left(p \vee \square_{\mathrm{F}} q \vee \square_{\mathrm{P}} p \vee r\right)$ & 1 & 1 & 1 & 1 & 1 & 1 & 1 & 1 \\
\hline
\end{tabular}

Table 3 Considered normal forms

\begin{tabular}{lll}
\hline Class & Description & Restrictions on $n, m$ \\
\hline CNF & No restrictions on (2) & - \\
HORN & At most one positive temporal literal & $m \leq 1$ \\
KROM & Binary clauses & $n+m \leq 2$ \\
\hline
\end{tabular}

Restrictions refer to Eq. (2)

Note that the operator name $G$ instead of $\square_{\mathrm{F}}$ often occurs in literature. Yet, in contrast to $\mathrm{G} \varphi$, for $\square_{\mathrm{F}} \varphi$ it is not required that $\varphi$ holds in the present world. We distinguish fragments of LTL by adding superscripts and subscripts as fol-

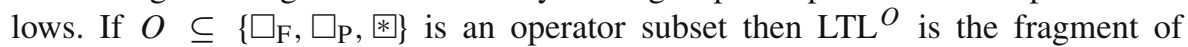
LTL consisting of formulas that are allowed to only use temporal operators from $O$ for temporal literals, i.e., it is a constraint on the allowed operators in equation (1) from above. We also consider restrictions of the clausal normal form in (2): $\circledast\left(\neg \lambda_{1} \vee \cdots \vee \neg \lambda_{n} \vee \lambda_{n+1} \vee \cdots \lambda_{n+m}\right)$. Table 3 lists the relevant cases for this study. If $\alpha \in$ \{CNF, HORN, KROM $\}$ then $\mathrm{LTL}_{\alpha}$ is the set of formulas where the subformulas of the type $\Theta\left(\neg \lambda_{1} \vee \cdots \vee \neg \lambda_{n} \vee \lambda_{n+1} \vee \cdots \lambda_{n+m}\right)$ (3), obey the normal form $\alpha$.

The following lemma shows a log-space constructible normal form which prohibits deep nesting of temporal operators of the investigated formulas.

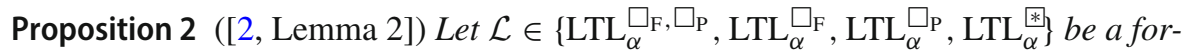
mula class for $\alpha \in\{\mathrm{CNF}, \mathrm{HORN}, \mathrm{KROM}\}$. For any formula $\varphi \in \mathcal{L}$, one can construct, in log-space, a satisfiability-equivalent $\mathcal{L}$-formula $\Psi \wedge$ 困 $\Phi$, where $\Psi$ is a conjunction of propositional variables from $\Phi$, and $\Phi$ is a conjunction of clauses of the form (3) containing only $\square_{\mathrm{F}}, \square \mathrm{P}$ for $\mathrm{LTL}_{\alpha} \square_{\mathrm{F}}, \square_{\mathrm{P}}, \square_{\mathrm{F}}$ for $\mathrm{LTL}_{\alpha} \square_{\mathrm{F}}, \square \mathrm{P}$ for $\mathrm{LTL}_{\alpha} \square_{\mathrm{P}}$, and only 米 for $\mathrm{LTL}_{\alpha}^{*}$, in which the temporal operators are not nested.

In the following sections we consider only formulas given in this normal form $\Psi \wedge$ $\Psi$. 


\section{Introduction of Backdoors for the Global Fragment of LTL}

In the following, we will introduce a notion of backdoors for formulas in the global fragment of linear temporal logic. The definition of these backdoors turns out to be very similar to the definition of the so-called strong backdoor sets for propositional formulas [18]. The main difference is that whenever a propositional variable is in the backdoor set then also all of its temporal literals are required to be in the backdoor set as well. A consequence of this is that in contrast to propositional formulas, where a backdoor set needs to consider all assignments of the backdoor set variables, we only need to consider assignments that are consistent between propositional variables and their temporal literals.

Let $\mathcal{O}$ be a set of operators. An assignment $\theta: \operatorname{Vars}(\phi) \cup\{O x \mid x \in \operatorname{Vars}(\phi) \wedge O \in$ $\mathcal{O}\} \rightarrow\{0,1\}$ is consistent if for every $x \in \operatorname{Vars}(\phi)$ it holds that if $\theta\left(\vartheta^{*} x\right)=1$, then also $\theta\left(\square_{P} x\right)=1, \theta\left(\square_{F} x\right)=1$, and $\theta(x)=1$.

Definition 3 (Backdoors) Let $\mathcal{C}$ be a class of CNF-formulas, $\mathcal{O}$ be a set of operators, and $\phi$ be an $\mathrm{LTL}_{\mathrm{CNF}}^{\mathcal{O}}$ formula. A set $X \subseteq \operatorname{Vars}(\phi)$ is a (strong) $(\mathcal{C}, \mathcal{O})$-backdoor if for every consistent assignment $\theta: X \cup\{O x \mid x \in X, O \in \mathcal{O}\} \rightarrow\{0,1\}$ it holds that $\phi[\theta]$ is in $\mathcal{C}$.

The reduct $\phi[\theta]$ is defined similarly to that for standard CNF-formulas, i.e., all clauses that contain a satisfied literal are deleted, and all falsified literals are deleted from their clauses. Here empty clauses are substituted by false, and the empty formula by true. Sometimes if the context of $\mathcal{O}$ is clear, we omit to state it and just mention the backdoor class $\mathcal{C}$.

Example 4 Let $\varphi=p_{1} \wedge p_{2} \wedge \circledast\left(\neg \square \mathrm{P} p_{4} \vee \square \mathrm{P} p_{2} \vee \square \mathrm{P} p_{3}\right)$ be the considered formula. Then $B=\left\{p_{3}\right\}$ is a strong (KROM, $\left\{\square_{\mathrm{P}}, \circledast\right\}$ )-backdoor as the following assignments have to be examined:

\begin{tabular}{|c|c|c|c|c|}
\hline$p_{3}$ & $\square \mathrm{P} p_{3}$ & $\circledast p_{3}$ & $\varphi[\theta]$ & \\
\hline 0 & 0 & 0 & $p_{1} \wedge p_{2} \wedge$ $*\left(\neg \square \mathrm{p} p_{4} \vee \square \mathrm{p} p_{2}\right)$ & 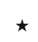 \\
\hline 0 & 0 & 1 & Irrelevant as inconsistent & \\
\hline 0 & 1 & 0 & $p_{1} \wedge p_{2}$ & 0 \\
\hline 0 & 1 & 1 & Irrelevant as inconsistent & \\
\hline 1 & 0 & 0 & 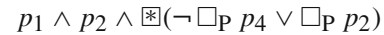 & $\star$ \\
\hline 1 & 0 & 1 & Irrelevant as inconsistent & \\
\hline 1 & 1 & 0 & $p_{1} \wedge p_{2}$ & Q \\
\hline 1 & 1 & 1 & $p_{1} \wedge p_{2}$ & \\
\hline
\end{tabular}

First, observe that all relevant rows lead to a KROM-formula. Note that for the rows marked with $\star$ the reduct just removed the temporal literal $\square_{\mathrm{P}} p_{3}$. All other rows are either inconsistent (and hence irrelevant) or delete the clause ( $\neg \square_{\mathrm{P}} p_{4} \vee \square \mathrm{P} p_{2} \vee$ $\left.\square_{\mathrm{P}} p_{3}\right)$ completely, because $\square_{\mathrm{P}} p_{3}$ is set to true. At first glance, our definition of backdoor sets for LTL is almost purely syntactical, and thereby is an accordance to 
strong backdoor sets for the propositional satisfiability problem. For instance consider the assignments marked with the $\odot$. In these cases we delete the clause $\left(\neg \square\right.$ P $p_{4} \vee$ $\left.\square_{\mathrm{P}} p_{2} \vee \square_{\mathrm{P}} p_{3}\right)$ completely because $\square_{\mathrm{P}} p_{3}$ is set to true. However, we also know that, because $* p_{3}$ is set to false, the clause will not be satisfied solely by $\square_{\mathrm{P}} p_{3}$ in all possible worlds of a satisfying model. This indicates that solving the formula using the backdoor will not be as simple as it was for the propositional satisfiability problem, where it was sufficient to enumerate all assignments of the backdoor set and solve the reduced formula. Nevertheless, as we will show in Sect. 5.1 our backdoor sets can still be used for the efficient evaluation of LTL formulas.

To exploit backdoor sets to obtain efficient fpt-algorithms for LTL one needs to accomplish two tasks: first, one needs to find a small backdoor set, and then one needs to show how the backdoor set can be exploited to efficiently evaluate the formula. This leads to the following problem definitions for every class $\mathcal{C}$ of formulas and set of operators $\mathcal{O}$.

Problem: $\quad \operatorname{Eval}^{\mathcal{O}}(\mathcal{C})-$ Backdoor evaluation to $\operatorname{LTL}_{\mathcal{C}}^{\mathcal{O}}$.

Input: $\quad \operatorname{LTL}_{\mathrm{CNF}}^{\mathcal{O}}$ formula $\phi$, strong $(\mathcal{C}, \mathcal{O})$-backdoor $X$.

Parameter: $|X|$.

Question: Is $\phi$ satisfiable?

Problem: $\quad \operatorname{Detect}^{\mathcal{O}}(\mathcal{C})$ - Backdoor detection to $\operatorname{LTL}_{\mathcal{C}}^{\mathcal{O}}$.

Input: $\quad \operatorname{LTL}_{\mathrm{CNF}}^{\mathcal{O}}$ formula $\phi$, integer $k \in \mathbb{N}$.

Parameter: $k$.

Task: $\quad$ Find a strong $(\mathcal{C}, \mathcal{O})$-backdoor of size $\leq k$ if one exists.

Of course, this approach is only meaningful if one considers target classes that have polynomial time solvable satisfiability problems. Artale et al. have shown [2] that satisfiability for LTL ${ }_{\text {HORN }}^{\circledast}$ and LTL ${ }_{\text {KROM }}^{\circledR}$ are solvable in P. Adding $\square_{\mathrm{F}}, \square_{\mathrm{P}}$ to the set of allowed operators makes the KROM fragment NP-complete whereas for HORN formulas the problem stays in P. Accordingly, we will consider in the following only KROM and HORN formulas. Moreover, note that when considering arbitrary CNF formulas instead of HORN or KROM formulas, then $\mathrm{LTL}_{\mathrm{CNF}}^{\mathcal{O}}$ is known to be NP-complete for any (even empty) subset $\mathcal{O} \subseteq\left\{\square_{\mathrm{F}}, \square_{\mathrm{P}}\right.$, $*$ \} [2].

\section{Backdoor Set Detection}

In this section, we show that finding strong $\mathcal{C}$-backdoor sets (under the parameter size of the set) is fixed-parameter tractable if $\mathcal{C}$ is either HORN or KROM. The algorithms that we will present are very similar to the algorithms that are known for the detection of strong backdoors for propositional CNF formulas [18].

We first show how to deal with the fact that we only need to consider consistent assignments. The following observation is easily witnessed by the fact that if one of

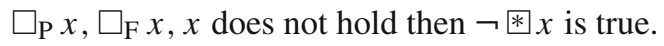

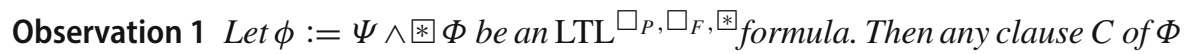
containing $\neg$ * $x$ and (at least) one of $\square_{P} x, \square_{F} x$ or $x$ for some variable $x \in \operatorname{Vars}(\phi)$ $i$ tautological and can be removed from $\Phi$ (without changing the satisfiability of $\phi$ ). 
Observe that the tautological clauses above are exactly the clauses that are satisfied by every consistent assignment. It follows that once these clauses are removed from the formula, it holds that for every clause $C$ of $\phi$ there is a consistent assignment $\theta$ such that $C$ is not satisfied by $\theta$.

Theorem 5 For every $\mathcal{O} \subseteq\left\{\right.$ 困, $\left.\square_{P}, \square_{F}\right\}$ and $\mathcal{C} \in\{$ HORN, KROM $\}$ the problem Detect $^{\mathcal{O}}(\mathcal{C})$ is in FPT.

Proof Let $\mathcal{O} \subseteq\left\{\right.$ 舟, $\left.\square_{P}, \square_{F}\right\}$. We will reduce Detect $^{\mathcal{O}}$ (HORN) to the problem VertexCover which is well-known to be fixed-parameter tractable (parameterized by the solution size) and which can actually be solved very efficiently in time $O\left(1.2738^{k}+k n\right)$ [6], where $k$ is the size of the vertex cover and $n$ the number of vertices in the input graph. Recall that given an undirected graph $G$ and an integer $k$, VertexCover asks whether there is a subset $C \subseteq V(G)$ of size at most $k$ (which is called a vertex cover of $G$ ) such that $C \cap e \neq \emptyset$ for every $e \in E(G)$. Given an LTL $\mathcal{O}$ formula $\phi:=\Psi \wedge$ $:$, we will construct an undirected graph $G$ such that $\phi$ has a strong HORN-backdoor of size at most $k$ if and only if $G$ has a vertex cover of size at most $k$. The graph $G$ has vertex set $\operatorname{Vars}(\phi)$ and there is an edge between two vertices $x$ and $y$ in $G$ if and only if there is a clause that contains at least two literals from $\{x, y\} \cup\{O x, O y \mid O \in \mathcal{O}\}$. Note that if $x=y$, the graph $G$ contains a self-loop. We claim that a set $X \subseteq \operatorname{Vars}(\phi)$ is a strong HORN-backdoor if and only if $X$ is a vertex cover of $G$.

Towards showing the forward direction, let $X \subseteq \operatorname{Vars}(\phi)$ be a strong HORNbackdoor set of $\phi$. We claim that $X$ is also a vertex cover of $G$. Suppose for a contradiction that $X$ is not a vertex cover of $G$, i.e., there is an edge $\{x, y\} \in E(G)$ such that $X \cap\{x, y\}=\emptyset$. Because $\{x, y\} \in E(G)$, we obtain that there is a clause $C$ in $\Phi$ that contains at least two literals from $\{x, y\} \cup\{O x, O y \mid O \in \mathcal{O}\}$. Moreover, because of Observation 1 there is a consistent assignment $\theta: X \cup\{O x \mid x \in X \wedge O \in \mathcal{O}\} \rightarrow\{0,1\}$ that falsifies all literals of $C$ over variables in $X$. Consequently, $\phi[\theta]$ contains a subclause of $C$ that still contains at least two literals from $\{x, y\} \cup\{O x, O y \mid O \in \mathcal{O}\}$. As a reason for this, $\phi[\theta] \notin \mathrm{HORN}$, contradicting our assumption that $X$ is a strong HORN-backdoor set of $\phi$.

Towards showing the reverse direction, let $X \subseteq V(G)$ be a vertex cover of $G$. We claim that $X$ is also a strong HORN-backdoor of $\phi$. Suppose for a contradiction that this is not the case, then there is an (consistent) assignment $\theta: X \cup\{O x \mid x \in X \wedge O \in$ $\mathcal{O}\} \rightarrow\{0,1\}$ and a clause $C$ in $\phi[\theta]$ containing two positive literals say over variables $x$ and $y$. We obtain that $C$ contains at least two positive literals from $\{x, y\} \cup\{O x, O y \mid$ $O \in \mathcal{O}\}$ and consequently $G$ contains the edge $\{x, y\}$, contradicting our assumption that $X$ is a vertex cover of $G$.

Now we will reduce Detect ${ }^{\mathcal{O}}$ (KROM) to the 3 -HittingSet problem, which is wellknown to be fixed-parameter tractable (parameterized by the solution size) [1]. Recall that given a universe $U$, a family $\mathcal{F}$ of subsets of $U$ of size at most three, and an integer $k$, 3-HittingSet asks whether there is a subset $S \subseteq U$ of size at most $k$ (which is called a hitting set of $\mathcal{F}$ ) such that $S \cap F \neq \varnothing$ for every $F \in \mathcal{F}$. Given an LTL ${ }^{\mathcal{O}}$ formula $\phi:=\Psi \wedge ⿴ \Phi$, we will construct a family $\mathcal{F}$ of subsets (of size at most three) of a universe $U$ such that $\phi$ has a strong KROM-backdoor of size at most $k$ if and only if $\mathcal{F}$ has a hitting set of size at most $k$. The universe $U$ is equal to $\operatorname{Vars}(\phi)$ and $\mathcal{F}$ contains 
the set $\operatorname{Vars}(C)$ for every set $C$ of exactly three literals contained in some clause of $\Phi$. We claim that a set $X \subseteq \operatorname{Vars}(\phi)$ is a strong KROM-backdoor if and only if $X$ is a hitting set of $\mathcal{F}$.

Towards showing the forward direction, let $X \subseteq \operatorname{Vars}(\phi)$ be a strong KROMbackdoor set of $\phi$ and suppose for a contradiction that there is a set $F \in \mathcal{F}$ such that $X \cap F=\emptyset$. It follows from the construction of $\mathcal{F}$ that $\Phi$ contains a clause $C$ containing at least three literals over the variables in $F$. Moreover, because of Observation 1 there is a consistent assignment $\theta: X \cup\{O x \mid x \in X \wedge O \in \mathcal{O}\} \rightarrow\{0,1\}$ that falsifies all literals of $C$ over variables in $X$. Consequently, $\phi[\theta]$ contains a subclause of $C$ that still contains at least three literals over the variables in $F$. As a result, $\phi[\theta] \notin \mathrm{KROM}$, contradicting our assumption that $X$ is a strong KROM-backdoor set of $\phi$.

Towards showing the reverse direction, let $X \subseteq U$ be a hitting set of $\mathcal{F}$ and suppose for contradiction that there is an (consistent) assignment $\theta: X \cup\{O x \mid x \in X \wedge O \in$ $\mathcal{O}\} \rightarrow\{0,1\}$ and a clause $C$ in $\phi[\theta]$ containing at least three literals. Let $C^{\prime}$ be a set of at exactly three literals from $C$. It follows from the construction of $\mathcal{F}$, that $\mathcal{F}$ contains the set $\operatorname{Vars}\left(C^{\prime}\right)$, however, $\operatorname{Vars}\left(C^{\prime}\right) \cap X=\emptyset$ contradicting our assumption that $X$ is a hitting set of $G$.

Having shown that the detection problem is fixed-parameter tractable, we now proceed to the backdoor set evaluation problem. We begin by investigating this problem for the class HORN and show that the problem lies in FPT.

\section{Backdoor Set Evaluation}

\subsection{Formulas Using only the Always Operator}

We showed in the previous section that strong backdoors can be found to the classes HORN and KROM in FPT time. In fact, this result holds independently of the considered temporal operators. In this section, we will consider the question of efficiently using a backdoor set to decide the satisfiability of a formula in the case of formulas restricted to the $*$ operator. We will show that this problem is in FPT for the class of HORN formulas but not for KROM formulas. Our fixed-parameter tractability result for HORN formulas largely depends on the special semantics of formulas restricted to the $*$ operators. Consequently, we will start by stating some properties of these formulas necessary to obtain our tractability result.

Let $\mathfrak{M}=(\mathbb{Z},<, V)$ be a temporal interpretation. We denote by $\operatorname{Vars}(\mathfrak{M})$ the set of propositions (in the following referred to as variables) for which $V$ is defined. For a set of variables $X \subseteq \operatorname{Vars}(\mathfrak{M})$, we denote by $\mathfrak{M}_{\mid X}$ the projection of $\mathfrak{M}$ onto $X$, i.e., the temporal interpretation $\mathfrak{M}_{\mid X}=\left(\mathbb{Z},<, V_{\mid X}\right)$, where $V_{\mid X}$ is only defined for the variables in $X$ and $V_{\mid X}(x)=V(x)$ for every $x \in X$. For an integer $z$, we denote by $\mathbf{A}(\mathfrak{M}, z)$ the assignment $\theta: \operatorname{Vars}(\mathfrak{M}) \rightarrow\{0,1\}$ holding at world $z$ in $\mathfrak{M}$, i.e., $\theta(v)=1$ if and only if $z \in \mathfrak{M}(v)$ for every $v \in \operatorname{Vars}(\mathfrak{M})$. Moreover, for a set of worlds $Z \subseteq \mathbb{Z}$ we denote by $\mathbf{A}(\mathfrak{M}, Z)$ the set of all assignments occurring in some world in $Z$ of $\mathfrak{M}$, i.e., $\mathbf{A}(\mathfrak{M}, Z):=\{\mathbf{A}(\mathfrak{M}, z) \mid z \in Z\}$. We also set $\mathbf{A}(\mathfrak{M})$ to be $\mathbf{A}(\mathfrak{M}, \mathbb{Z})$. For an 
Table 4 An example for the notions $\mathbf{G}(\mathbb{A}, V)$ and $\mathbf{G}(\mathbb{A}, V, \theta)$

\begin{tabular}{llllllll}
\hline & $\mathbb{A}$ & & & & $\theta$ & $\mathbf{G}(\mathbb{A}, V)\left(\mathbb{*} v_{i}\right)$ & $\mathbf{G}(\mathbb{A}, V, \theta)\left(v_{i}\right)$ \\
\cline { 2 - 5 }$\alpha_{1}$ & $\alpha_{2}$ & $\alpha_{3}$ & $\alpha_{4}$ & & & 1 \\
\hline$v_{1}$ & 0 & 1 & 0 & 1 & 1 & 0 & 0 \\
$v_{2}$ & 1 & 1 & 1 & 1 & 0 & 1 & 1 \\
$v_{3}$ & 1 & 1 & 0 & 0 & 1 & 0 & 0 \\
$v_{4}$ & 1 & 1 & 1 & 1 & 0 & 1 & \\
\hline
\end{tabular}

assignment $\theta: X \rightarrow\{0,1\}$, we denote by $\mathbf{W}(\mathfrak{M}, \theta)$ the set of all worlds $z \in \mathbb{Z}$ of $\mathfrak{M}$ such that $\mathbf{A}(\mathfrak{M}, z)$ is equal to $\theta$ on all variables in $X$.

Let $\varphi:=\Psi \wedge \circledast \Phi \in \mathrm{LTL}_{\mathrm{CNF}}^{*}$. We denote by $\mathbf{C N F}(\Phi)$ the propositional CNF formula obtained from $\Phi$ after replacing each occurrence of $⿴ x$ in $\Phi$ with the same fresh propositional variable (with the same name). For instance, $* a \wedge \circledast a$ is replaced by $⿴ 囗 ⿱ 一 一 \wedge ⿴ 囗 十$, where $⿴ a$ is a fresh propositional variable. For a set of variables $V$ and a set of assignments $\mathbb{A}$ of the variables in $V$, we denote by $\mathbf{G}(\mathbb{A}, V):\{\circledast v \mid v \in V\} \rightarrow$ $\{0,1\}$ the assignment defined by setting $\mathbf{G}(\mathbb{A}, V)(* v)=1$ if and only if $\alpha(v)=1$ for every $\alpha \in \mathbb{A}$. Moreover, if $\theta: V \rightarrow\{0,1\}$ is an assignment of the variables in $V$, we denote by $\mathbf{G}(\mathbb{A}, V, \theta)$ the assignment defined by setting $\mathbf{G}(\mathbb{A}, V, \theta)(v)=\theta(v)$ and $\mathbf{G}(\mathbb{A}, V, \theta)(\circledast v)=\mathbf{G}(\mathbb{A}, V)(* v)$ for every $v \in V$. An example for these notions is given in Table 4. For a set $\mathbb{A}$ of assignments over $V$ and an assignment $\theta: V^{\prime} \rightarrow\{0,1\}$ with $V^{\prime} \subseteq V$, we denote by $\mathbb{A}(\theta)$ the set of all assignments $\alpha \in \mathbb{A}$ such that $\alpha(v)=$ $\theta(v)$ for every $v \in V^{\prime}$.

For a set $\mathbb{A}$ of assignments over some variables $V$ and a subset $V^{\prime} \subseteq V$, we denote by $\left.\mathbb{A}\right|_{V^{\prime}}$ the projection of $\mathbb{A}$ onto $V^{\prime}$, i.e., the set of assignments $\alpha \in \mathbb{A}$ restricted to the variables in $V^{\prime}$.

Intuitively the next lemma describes the translation of a temporal model into separate satisfiability checks for propositional formulas.

Lemma 6 Let $\varphi:=\Psi \wedge$ $⿴ 囗 \Psi \in \mathrm{LTL}^{*}$. Then, $\varphi$ is satisfiable if and only if there is a set $\mathbb{A}$ of assignments of the variables in $\varphi$ and an assignment $\alpha_{0} \in \mathbb{A}$ such that: $\alpha_{0}$ satisfies $\Psi$ and for every assignment $\alpha \in \mathbb{A}$ it holds that $\mathbf{G}(\mathbb{A}, \operatorname{Vars}(\varphi), \alpha)$ satisfies the propositional formula $\mathbf{C N F}(\Phi)$.

Proof Towards showing the forward direction assume that $\varphi:=\Psi \wedge \circledast \Phi$ is satisfiable and let $\mathfrak{M}$ be a temporal interpretation witnessing this. It is easy to check from the definition that the set of assignments $\mathbb{A}:=\mathbf{A}(\mathfrak{M})$ together with the assignment $\alpha_{0}:=$ $\mathbf{A}(\mathfrak{M}, 0)$ satisfy the conditions of the lemma.

Towards showing the reverse direction assume that $\mathbb{A}:=\left\{\alpha_{0}, \ldots, \alpha_{|\mathbb{A}|-1}\right\}$ is as given in the statement of the lemma. We claim that the temporal interpretation $\mathfrak{M}$ defined below satisfies the formula $\varphi$. Let $\mathbb{Z}_{<0}$ be the set of all integers smaller than 0 and let $\mathbb{Z}_{>|\mathbb{A}|}$ be the set of all integers greater than $|\mathbb{A}|$. Then for every variable $v \in \operatorname{Vars}(\varphi)$, the set $\mathfrak{M}(v)$ contains the set $\left\{z\left|\alpha_{z}(v)=1 \wedge 0 \leq z \leq\right| \mathbb{A} \mid\right\}$. Moreover, if $\alpha_{0}(v)=1, \mathfrak{M}(v)$ also contains the set $\mathbb{Z}_{<0}$ and if $\alpha_{|\mathbb{A}|}(v)=1, \mathfrak{M}(v)$ additionally contains the set $\mathbb{Z}_{>|\mathbb{A}|}$. It is easy to verify that $\mathfrak{M}, 0 \models \varphi$. 
Informally, the following lemma shows that for deciding the satisfiability of an LTL $^{*}$ formula, we only need to consider sets of assignments $\mathbb{A}$, whose size is linear (instead of exponential) in the number of variables.

Lemma 7 Let $\varphi:=\Psi \wedge \circledast \Phi \in \mathrm{LTL}^{\circledast}$ and $X \subseteq \operatorname{Vars}(\varphi)$. Then $\varphi$ is satisfiable if and only if there is a set $\Theta$ of assignments of the variables in $X$, an assignment $\theta_{0} \in \Theta$, a set $\mathbb{A}$ of assignments of the variables in $\operatorname{Vars}(\varphi)$, and an assignment $\alpha_{0} \in \mathbb{A}$ such that:

(C1) the set $\Theta$ is equal to $\mathbb{A}_{\mid X}$,

(C2) the assignment $\theta_{0}$ is equal to $\left.\alpha_{0}\right|_{X}$,

(C3) $\mathbb{A}$ and $\alpha_{0}$ satisfy the conditions stated in Lemma 6, and

(C4) $|\mathbb{A}(\theta)| \leq|\operatorname{Vars}(\varphi) \backslash X|+1$ for every $\theta \in \Theta$.

Proof Note that the reverse direction follows immediately from Lemma 6, because the existence of the set of assignments $\mathbb{A}$ and the assignment $\alpha_{0}$ satisfying condition (C3) imply the satisfiability of $\varphi$.

Towards showing the forward direction assume that $\varphi$ is satisfiable. Because of Lemma 6 there is a set $\mathbb{A}$ of assignments of the variables in $\varphi$ and an assignment $\alpha_{0} \in \mathbb{A}$ that satisfy the conditions of Lemma 6 . Let $\Theta$ be equal to $\mathbb{A}_{\mid X}$ and $\theta_{0}$ be equal to $\left.\alpha_{0}\right|_{X}$. Observe that setting $\Theta$ and $\theta_{0}$ in this way already satisfies $(\mathrm{C} 1)$ to $(\mathrm{C} 3)$. We will show that there is a subset of $\mathbb{A}$ that still satisfies (C1)-(C3) and additionally (C4). Towards showing this consider any subset $\mathbb{A}^{\prime}$ of $\mathbb{A}$ that satisfies the following three conditions: (1) $\alpha_{0} \in \mathbb{A}^{\prime}$, (2) for every $\theta \in \Theta$ it holds that $\mathbb{A}^{\prime}(\theta) \neq \emptyset$, and (3) for every variable $v$ of $\varphi$ and every $b \in\{0,1\}$ it holds that there is an assignment $\alpha \in \mathbb{A}$ with $\alpha(v)=i$ if and only if there is an assignment $\alpha^{\prime} \in \mathbb{A}^{\prime}$ with $\alpha^{\prime}(v)=i$. Note that conditions (1) and (2) ensure that $\mathbb{A}^{\prime}$ satisfies (C1) and (C2) and condition (3) ensures (C3). Accordingly, any subset $\mathbb{A}^{\prime}$ satisfying conditions (1)-(3) still satisfies (C1)-(C3). It remains to show how to obtain such a subset $\mathbb{A}^{\prime}$ that additionally satisfies $(\mathrm{C} 4)$. We define $\mathbb{A}^{\prime}$ as follows. Let $\mathbb{A}_{0}^{\prime}$ be a subset of $\mathbb{A}$ containing $\alpha_{0}$ as well as one arbitrary assignment $\alpha \in \mathbb{A}(\theta)$ for every $\theta \in \Theta$. Note that $\mathbb{A}_{0}^{\prime}$ already satisfies conditions (1) and (2) as well as condition (3) for every variable $v \in X$. Observe furthermore that if there is a variable $v$ of $\varphi$ such that condition (3) is violated by $\mathbb{A}_{0}^{\prime}$ then it is sufficient to add at most one additional assignment to $\mathbb{A}_{0}^{\prime}$ in order to satisfy condition (3) for $v$. Let $\mathbb{A}^{\prime}$ be obtained from $\mathbb{A}_{0}^{\prime}$ by adding (at most $\left.|\operatorname{Vars}(\varphi) \backslash X|\right)$ assignments in order to ensure condition (3) for every variable $v \in \operatorname{Vars}(\varphi) \backslash X$. Then $\mathbb{A}^{\prime}$ satisfies the conditions of the lemma.

We are now ready to show the tractability of the evaluation of strong HORN-backdoor sets.

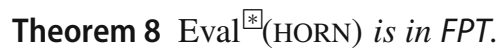

Proof Let $\varphi:=\Psi \wedge \circledast \Phi \in \mathrm{LTL}^{*}$ and let $X \subseteq \operatorname{Vars}(\varphi)$ be a strong HORN-backdoor of $\varphi$. The main idea of the algorithm is as follows: For every set $\Theta$ of assignments of the variables in $X$ and every $\theta_{0} \in \Theta$, we will construct a propositional HORN-formula $F_{\Theta, \theta_{0}}$, which is satisfiable if and only if there is a set $\mathbb{A}$ of assignments of the variables in $\operatorname{Vars}(\varphi)$ and an assignment $\alpha_{0} \in \mathbb{A}$ satisfying the conditions of Lemma 7 . It then 
follows from Lemma 7 that $\varphi$ is satisfiable if and only if there is such a set $\Theta$ of assignments and an assignment $\theta_{0} \in \Theta$ for which $F_{\Theta, \theta_{0}}$ is satisfiable. Because there are at most $2^{2^{|X|}}$ such sets $\Theta$ and at most $2^{|X|}$ such assignments $\theta_{0}$ and for each of these sets the formula $F_{\Theta, \theta_{0}}$ is a HORN-formula, it follows that checking whether there are $\Theta$ and $\theta_{0}$ such that the formula $F_{\Theta, \theta_{0}}$ is satisfied (and as a result decide the satisfiability of $\varphi)$ can be done in time $O\left(2^{2^{|X|}} \cdot 2^{|X|} \cdot\left|F_{\Theta, \theta_{0}}\right|\right)$. Since we will show below that the length of the formula $F_{\Theta, \theta_{0}}$ can be bounded by an (exponential) function of $|X|$ times a polynomial in the input size, i.e., the length of the formula $\varphi$, this implies that $\mathrm{Eval}^{*}$ (HORN) is in FPT.

The remainder of the proof is devoted to the construction of the formula $F_{\Theta, \theta_{0}}$ for a fixed set of assignments $\Theta$ and a fixed assignment $\theta_{0} \in \Theta$ (and to show that it enforces the conditions of Lemma 7).

Let $R:=\operatorname{Vars}(\varphi) \backslash X$ and $r:=|R|+1$. For a propositional formula $F$, a subset $V \subseteq$ $\operatorname{Vars}(F)$, an integer $i$ and a label $s$, we denote by $\operatorname{copy}(F, V, i, s)$ the propositional formula obtained from $F$ after replacing each occurrence of a variable $v \in V$ with a novel variable $v_{s}^{i}$. We need the following auxiliary formulas. For every $\theta \in \Theta \backslash\left\{\theta_{0}\right\}$, let $F_{\Theta, \theta_{0}}^{\Theta}$ be the formula (where the notation $\Phi[\mathbf{G}(\Theta, X, \theta)]$ refers to the formula that is obtained after applying the assignment $\mathbf{G}(\Theta, X, \theta)$ in the usual sense, that is, removing satisfied clauses and deleting falsified literals):

\section{$\bigwedge_{1 \leq i \leq r} \operatorname{copy}(\mathbf{C N F}(\Phi[\mathbf{G}(\Theta, X, \theta)]), R, i, \theta)$.}

Moreover, let $F_{\Theta, \theta_{0}}^{\theta_{0}}$ be the formula:

$$
\begin{array}{r}
\operatorname{copy}\left(\Psi\left[\theta_{0}\right] \wedge \mathbf{C N F}\left(\Phi\left[\mathbf{G}\left(\Theta, X, \theta_{0}\right)\right]\right), R, 1, \theta_{0}\right) \wedge \\
\bigwedge_{2 \leq i \leq r} \operatorname{copy}\left(\mathbf{C N F}\left(\Phi\left[\mathbf{G}\left(\Theta, X, \theta_{0}\right)\right]\right), R, i, \theta_{0}\right) .
\end{array}
$$

Observe that because $X$ is a strong HORN-backdoor set (and the formula $\Psi$ only consists of unit clauses), it holds that the formula $F_{\Theta, \theta_{0}}^{\theta}$ is HORN for every $\theta \in \Theta$. We also need the propositional formula $F_{\text {cons }}$ that enforces the consistency between the propositional variables $* x$ and the variables in $\left\{x_{\theta}^{i} \mid \theta \in \Theta \wedge 1 \leq i \leq r\right\}$ for every $x \in \operatorname{Vars}(\varphi) \backslash X$. The formula $F_{\text {cons }}$ consists of the following clauses: for every $\theta \in \Theta, i$ with $1 \leq i \leq r$, and $v \in R$, the clause $* v \rightarrow v_{\theta}^{i}=\neg \nLeftarrow v \vee v_{\theta}^{i}$ and for every $v \in R$ the clause

$$
\neg \circledast v \rightarrow \bigvee_{\theta \in \Theta \wedge 1 \leq i \leq r} \neg v_{\theta}^{i}=\circledast v \vee \bigvee_{\theta \in \Theta \wedge 1 \leq i \leq r} \neg v_{\theta}^{i}
$$

Observe that $F_{\text {cons }}$ is a HORN formula.

Finally the formula $F_{\Theta, \theta_{0}}$ is defined as: $\bigwedge_{\theta \in \Theta} F_{\Theta, \theta_{0}}^{\theta} \wedge F_{\text {cons }}$. 
Note that $F_{\Theta, \theta_{0}}$ is HORN and the length of $F_{\Theta, \theta_{0}}$ is at most

$$
\begin{aligned}
\left|F_{\Theta, \theta_{0}}\right| & \leq \sum_{\theta \in \Theta}\left|F_{\Theta, \theta_{0}}^{\Theta}\right|+\left|F_{\text {cons }}\right| \\
& \leq 2^{|X|}(|\operatorname{Vars}(\varphi) \backslash X|+1)(|\Phi|+|\Psi|)+2 \cdot 2^{|X|} \cdot(|\operatorname{Vars}(\varphi) \backslash X|+1)^{2}
\end{aligned}
$$

and consequently bounded by a function of $|X|$ times a polynomial in the input size. It is now relatively straightforward to verify that $F_{\Theta, \theta}$ is satisfiable if and only if there is a set $\mathbb{A}$ of assignments of the variables in $\operatorname{Vars}(\varphi)$ and an assignment $\alpha_{0} \in \mathbb{A}$ satisfying the conditions of Lemma 7. Informally, for every $\theta \in \Theta$, each of the $r$ copies of the formula $\mathbf{C N F}(\Phi[\mathbf{G}(\Theta, X, \theta)])$ represent one of the at most $r$ assignments in $\mathbb{A}(\theta)$, the formula $F_{\Theta, \theta_{0}}^{\theta_{0}}$ ensures (among other things) that the assignment chosen for $\alpha_{0}$ satisfies $\Psi$ and the formula $F_{\text {cons }}$ ensures that the "global assignments" represented by the propositional variables $⿴ x$ are consistent with the set of local assignments in $\mathbb{A}$ represented by the variables in $\left\{x_{\theta}^{i} \mid \theta \in \Theta \wedge 1 \leq i \leq r\right\}$ for every $x \in \operatorname{Vars}(\varphi) \backslash X$.

Surprisingly, the next result will show that KROM formulas turn out to be quite challenging. Backdoor set evaluation of this class of formulas is proved to be paraNPcomplete which witnesses an intractability degree in the parameterized sense.

Theorem $9 \mathrm{Eval}^{\circledR}(\mathrm{KROM})$ is paraNP-complete (the NP-completeness already holds for backdoor sets of size two).

Proof The membership in paraNP follows because the satisfiability of $\mathrm{LTL}_{\mathrm{CNF}}^{\otimes}$ can be decided in NP [2, Table 1].

We show paraNP-hardness of Eval ${ }^{*}(\mathrm{KROM})$ by giving a polynomial time reduction from the NP-hard problem $3 \mathrm{COL}$ to Eval ${ }^{\text {* }}(\mathrm{KROM})$ for backdoors of size two. In 3COL one asks whether a given input graph $G=(V, E)$ has a coloring $f: V(G) \rightarrow\{1,2,3\}$ of its vertices with at most three colors such that $f(v) \neq f(u)$ for every edge $\{u, v\}$

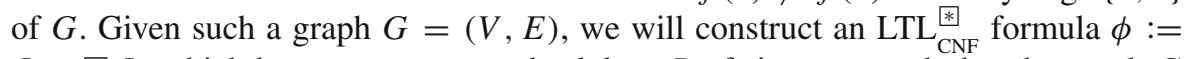
$\Psi \wedge \circledast \Phi$, which has a strong KROM-backdoor $B$ of size two, such that the graph $G$ has a 3-coloring if and only if $\phi$ is satisfiable.

For the remainder we will assume that there exists an arbitrary but fixed ordering of the vertices $V(G)=\left\{v_{1}, \ldots, v_{n}\right\}$. Further for the construction we assume w.l.o.g. that any undirected edge $e=\left\{v_{i}, v_{j}\right\} \in E$ follows this ordering, i.e., $i<j$. The formula $\phi$ contains the following variables:

(V1) The variables $b_{1}$ and $b_{2}$. These variables make up the backdoor set $B$, i.e., $B:=\left\{b_{1}, b_{2}\right\}$.

(V2) For every $i$ with $1 \leq i \leq n$, the variable $v_{i}$.

(V3) For every $e=\left\{v_{i}, v_{j}\right\} \in E(G)$ with $1 \leq i, j \leq n$ the variables $e_{i, j}^{b_{1} b_{2}}, e_{i, j}^{\bar{b}_{1} b_{2}}$, and $e_{i, j}^{b_{1} \bar{b}_{2}}$.

We set $\Psi$ to be the empty formula and the formula $\Phi$ contains the following clauses:

(C1) For every $i$ with $1 \leq i \leq n$, the clause $\neg$ * $v_{i}$. Informally, this clause ensures that $v_{i}$ has to be false at least at one world, which will later be used to assign a color to the vertex $v_{i}$ of $G$. Observe that the clause is KROM. 
Table 5 Given a graph $G=\left(\left\{v_{1}, v_{2}, v_{3}\right\},\left\{\left\{v_{1}, v_{2}\right\},\left\{v_{1}, v_{3}\right\},\left\{v_{2}, v_{3}\right\}\right\}\right)$ together with a 3-coloring $f\left(v_{i}\right)=$ $i$ for $1 \leq i \leq 3$, leads to the depicted temporal interpretation $\mathfrak{M}$ satisfying $\mathfrak{M} \models \phi$ given as a table

\begin{tabular}{ccccccccccccccc}
\hline & $b_{1}$ & $b_{2}$ & $v_{1}$ & $v_{2}$ & $v_{3}$ & $e_{1,2}^{b_{1} b_{2}}$ & $e_{1,2}^{\bar{b}_{1} b_{2}}$ & $e_{1,2}^{b_{1} \bar{b}_{2}}$ & $e_{1,3}^{b_{1} b_{2}}$ & $e_{1,3}^{\bar{b}_{1} b_{2}}$ & $e_{1,3}^{b_{1} \bar{b}_{2}}$ & $e_{2,3}^{b_{1} b_{2}}$ & $e_{2,3}^{\bar{b}_{1} b_{2}}$ & $e_{2,3}^{b_{1} \bar{b}_{2}}$ \\
\hline 1 & 0 & 0 & 0 & 1 & 1 & 1 & 0 & $*$ & 1 & $*$ & 0 & $*$ & 1 & 0 \\
2 & 1 & 0 & 1 & 0 & 1 & 1 & 0 & $*$ & 1 & $*$ & 0 & $*$ & 1 & 0 \\
3 & 0 & 1 & 1 & 1 & 0 & 1 & 0 & $*$ & 1 & $*$ & 0 & $*$ & 1 & 0
\end{tabular}

Each row of the table corresponds to a world as indicated by the first column of the table. Each column represents the assignments of a variable as indicated in the first row. A "*" indicates that the assignment is not fixed, i.e., the assignment does not influence whether $\mathfrak{M} \models \phi$

(C2) For every $e=\left\{v_{i}, v_{j}\right\} \in E(G)$ with $1 \leq i, j \leq n$, the clauses $v_{i} \vee$ $e_{i, j}^{b_{1} b_{2}} \vee$ $b_{1} \vee b_{2}, v_{i} \vee \circledast e_{i, j}^{\bar{b}_{1} b_{2}} \vee \neg b_{1} \vee b_{2}$, and $v_{i} \vee \circledast e_{i, j}^{b_{1} \bar{b}_{2}} \vee b_{1} \vee \neg b_{2}$ as well as the clauses $v_{j} \vee \neg \circledast e_{i, j}^{b_{1} b_{2}} \vee b_{1} \vee b_{2}, v_{j} \vee \neg \circledast e_{i, j}^{\bar{b}_{1} b_{2}} \vee \neg b_{1} \vee b_{2}$, and $v_{j} \vee \neg \circledast e_{i, j}^{b_{1} \bar{b}_{2}} \vee b_{1} \vee \neg b_{2}$. Observe that all of these clauses are KROM after deleting the variables in $B$.

(C3) The clause $\neg b_{1} \vee \neg b_{2}$. Informally, this clause excludes the color represented by setting $b_{1}$ and $b_{2}$ to true. Observe that the clause is KROM.

It follows from the definition of $\phi$ that $\phi[\theta] \in \mathrm{LTL}_{\text {KROM }}^{*}$ for every assignment $\theta$ of the variables in $B$. As a consequence, $B$ is a strong KROM-backdoor of size two of $\phi$ as required. Moreover, since $\phi$ can be constructed in polynomial time, it only remains to show that $G$ has a 3-coloring if and only if $\phi$ is satisfiable.

Towards showing the forward direction assume that $G$ has a 3-coloring and let $f: V(G) \rightarrow\{1,2,3\}$ be such a 3 -coloring for $G$. We will show that $\phi$ is satisfiable by constructing a temporal interpretation $\mathfrak{M}$ such that $\mathfrak{M} \models \phi$. The interpretation $\mathfrak{M}$ is defined as follows:

- For every $i$ with $1 \leq i \leq n$, we set $\mathfrak{M}\left(v_{i}\right)=\mathbb{Z} \backslash\left\{f\left(v_{i}\right)\right\}$.

- We set $\mathfrak{M}\left(b_{1}\right)=\{2\}$ and $\mathfrak{M}\left(b_{2}\right)=\{3\}$.

- For every $e=\left\{v_{i}, v_{j}\right\} \in E(G)$ :

- if $f\left(v_{i}\right)=1$ set $\mathfrak{M}\left(e_{i, j}^{b_{1} b_{2}}\right)=\mathbb{Z}$, else set $\mathfrak{M}\left(e_{i, j}^{b_{1} b_{2}}\right)=\emptyset$.

- if $f\left(v_{i}\right)=2$ set $\mathfrak{M}\left(e_{i, j}^{\bar{b}_{1} b_{2}}\right)=\mathbb{Z}$, else set $\mathfrak{M}\left(e_{i, j}^{\bar{b}_{1} b_{2}}\right)=\emptyset$.

- if $f\left(v_{i}\right)=3$ set $\mathfrak{M}\left(e_{i, j}^{b_{1} \bar{b}_{2}}\right)=\mathbb{Z}$, else set $\mathfrak{M}\left(e_{i, j}^{b_{1} \bar{b}_{2}}\right)=\varnothing$.

An example for such a temporal interpretation resulting for a simple graph is illustrated in Table 5. Towards showing that $\mathfrak{M} \models \phi$, we consider the different types of clauses given in (C1)-(C3).

- The clauses in (C1) hold because $\mathfrak{M}, f\left(v_{i}\right) \not \models v_{i}$ for every $i$ with $1 \leq i \leq n$.

- For every $e=\left\{v_{i}, v_{j}\right\} \in E(G)$, we have to show that the clauses given in (C2) are satisfied for every world. Because $f$ is a 3-coloring of $G$, we obtain that $f\left(v_{i}\right) \neq f\left(v_{j}\right)$. W.1.o.g. we assume in the following that $f\left(v_{i}\right)=1$ and $f\left(v_{j}\right)=2$. We first consider the clauses given in (C2) containing $v_{i}$. Because $\mathfrak{M}\left(v_{i}\right)=\mathbb{Z} \backslash\{1\}$, it only remains to consider the world 1 . In this world $b_{1}$ and $b_{2}$ are false. It follows that all clauses containing either $\neg b_{1}$ or $\neg b_{2}$ are satisfied in this world. As a reason 
for this, it only remains to consider clauses of the form $v_{i} \vee{ }^{b_{1} b_{2}} \vee b_{1} \vee b_{2}$. But these are satisfied because $f\left(v_{i}\right)=1$ implies that $\mathfrak{M}\left(e_{i, j}^{b_{1} b_{2}}\right)=\mathbb{Z}$.

Consider now the clauses given in (C2) that contain $v_{j}$. Using the same argumentation as used above for $v_{i}$, we obtain that we only need to consider world 2 and moreover we only need to consider clauses of the form $v_{j} \vee \neg \circledast e_{i, j}^{\bar{b}_{1} b_{2}} \vee \neg b_{1} \vee b_{2}$. Because $f\left(v_{i}\right)=1$, we obtain that $\mathfrak{M}\left(e_{i, j}^{\bar{b}_{1} b_{2}}\right)=\emptyset$, which implies that these clauses are also satisfied.

- The clause $\neg b_{1} \vee \neg b_{2}$ is trivially satisfied, because there is no world in which $b_{1}$ and $b_{2}$ hold simultaneously.

Towards showing the reverse direction assume that $\phi$ is satisfiable and let $\mathfrak{M}$ be a temporal interpretation witnessing this. First note that because of the clauses added by $\mathrm{C} 1$, it holds that $\mathfrak{M}\left(v_{i}\right) \neq \mathbb{Z}$ for every $i$ with $1 \leq i \leq n$. Let $w: V(G) \rightarrow \mathbb{Z}$ be defined such that for every $i$ with $1 \leq i \leq n, w\left(v_{i}\right)$ is an arbitrary world in $\mathbb{Z} \backslash \mathfrak{M}\left(v_{i}\right)$. We define $f: V(G) \rightarrow\{1,2,3\}$ by setting:

- $f\left(v_{i}\right)=1$ if $\mathfrak{M}, w\left(v_{i}\right) \not \models b_{1} \vee b_{2}$,

- $f\left(v_{i}\right)=2$ if $\mathfrak{M}, w\left(v_{i}\right) \not \models \neg b_{1} \vee b_{2}$, and

- $f\left(v_{i}\right)=3$ if $\mathfrak{M}, w\left(v_{i}\right) \not \models b_{1} \vee \neg b_{2}$.

Note that because of the clause added by (C3), $f$ assigns exactly one color to every vertex $v_{i}$ of $G$. We claim that $f$ is a 3-coloring of $G$. To show this it suffices to show that for every $e=\left\{v_{i}, v_{j}\right\} \in E(G)$, it holds that $f\left(v_{i}\right) \neq f\left(v_{j}\right)$. Assume for a contradiction that this is not the case, i.e., there is an edge $e=\left\{v_{i}, v_{j}\right\} \in E(G)$ such that $f\left(v_{i}\right)=f\left(v_{j}\right)$. W.l.o.g. assume furthermore that $f\left(v_{i}\right)=f\left(v_{j}\right)=1$. Consider the clause $v_{i} \vee \circledast e_{i, j}^{b_{1} b_{2}} \vee b_{1} \vee b_{2}$ (which was added by C2). Then, because of the definition of $w$ and $f$, we obtain that $\mathfrak{M}, w\left(v_{i}\right) \not \models v_{i} \vee b_{1} \vee b_{2}$. It follows that $\mathfrak{M}, w\left(v_{i}\right) \models \circledast e_{i, j}^{b_{1} b_{2}}$. Consider now the clause $v_{j} \vee \neg \circledast e_{i, j}^{b_{1} b_{2}} \vee b_{1} \vee b_{2}$ (which was added by $\mathrm{C} 2$ ). Then, again because of the choice of $w$ and $f$, we obtain that $\mathfrak{M}, w\left(v_{j}\right) \not v_{j} \vee b_{1} \vee b_{2}$. As a consequence, $\mathfrak{M}, w\left(v_{j}\right) \models \neg \circledast e_{i, j}^{b_{1} b_{2}}$ contradicting $\mathfrak{M}, w\left(v_{i}\right)=\circledast e_{i, j}^{b_{1} b_{2}}$. This completes the proof of the theorem.

\subsection{Globally in the Past and Globally in the Future}

Now we turn to a more flexible fragment where we can talk about the past as well as about the future and show it is possible to encode NP-complete problems into the HORN-fragment yielding a paraNP lower bound.

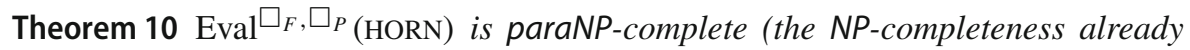
holds for backdoor sets of size four).

Proof The membership in paraNP follows as the satisfiability of $\mathrm{LTL}_{\mathrm{CNF}}^{\square_{F}, \square_{P}}$ can be decided in NP [2, Table 1].

We show paraNP-hardness of Eval $\square_{F}, \square_{P}$ (HORN) by describing a polynomial time

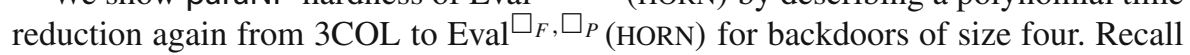
that in $3 \mathrm{COL}$ one asks whether a given input graph $G=(V, E)$ has a coloring 
$f: V(G) \rightarrow\{1,2,3\}$ of its vertices with at most three colors such that $f(v) \neq f(u)$ for every edge $\{u, v\}$ of $G$. Given such a graph $G=(V, E)$, we will construct an $\mathrm{LTL}_{\mathrm{CNF}}^{\square_{F}, \square_{P}}$ formula $\phi:=\Psi \wedge \circledast \Phi$, which has a strong HORN-backdoor $B$ of size four, such that the graph $G$ has a 3-coloring if and only if $\phi$ is satisfiable.

For the remainder we will assume that $V(G)=\left\{v_{1}, \ldots, v_{n}\right\}$ and $E(G)=$ $\left\{e_{1}, \ldots, e_{m}\right\}$. The formula $\phi$ contains the following variables:

(V1) The variables $c_{1}, c_{2}, c_{3}, p_{n}^{\prime}$. These variables make up the backdoor set $B$, i.e., $B:=\left\{c_{1}, c_{2}, c_{3}, p_{n}^{\prime}\right\}$.

(V2) The variable $s$, which indicates the starting world.

(V3) For every $i$ with $1 \leq i \leq n$, three variables $v_{i}^{1}, v_{i}^{2}, v_{i}^{3}$.

(V4) For every $i$ with $1 \leq i \leq n$ the variable $p_{i}$.

We set $\Psi$ to be the formula $s$ and the formula $\Phi$ contains the following clauses:

(C1) The clauses $c_{1} \vee c_{2} \vee c_{3}, \neg c_{1} \vee \neg c_{2} \vee \neg c_{3}, c_{1} \vee \neg c_{2} \vee \neg c_{3}, \neg c_{1} \vee \neg c_{2} \vee c_{3}$, and $\neg c_{1} \vee c_{2} \vee \neg c_{3}$. Informally, these clauses ensure that in every world it holds that exactly one of the variables $c_{1}, c_{2}, c_{3}$ is true. Note that $c_{1} \vee c_{2} \vee c_{3}$ is not HORN, however, all of its variables are contained in the backdoor set $B$.

(C2) For every $i$ and $c$ with $1 \leq i \leq n$ and $1 \leq c \leq 3$, the clauses $v_{i}^{c} \rightarrow \square_{F} v_{i}^{c}$ and $v_{i}^{c} \rightarrow \square_{P} v_{i}^{c}$; note that $v_{i}^{c} \rightarrow \square_{F} v_{i}^{c}$ corresponds to the clause $\neg v_{i}^{c} \vee \square_{F} v_{i}^{c}$. Informally, these clauses ensure that the variable $v_{i}^{c}$ either holds in every world or in no world for every $i$ and $c$ as above. Observe that both of these clauses are HORN.

(C3) Informally, the following set of clauses ensures together that for every $i$ with $1 \leq i \leq n$, it holds that $p_{i}$ is true in every world apart from the $i$-th world (where $p_{i}$ is false). Here, the first world is assumed to be the starting world.

(C3-1) The clauses $s \rightarrow \neg p_{1}, s \rightarrow \square_{F} p_{1}$, and $s \rightarrow \square_{P} p_{1}$. Informally, these ensure that $p_{1}$ is only false in the starting world (and otherwise true).

(C3-2) The clause $p_{i} \wedge \square_{F} p_{i} \rightarrow \square_{F} p_{i+1}$ for every $i$ with $1 \leq i<n$. Informally, these clauses (together with the clauses from C3-1) ensure that for every $i$ with $2 \leq i \leq n$, it holds that $p_{i}$ is true in every world after the $i$-th world.

(C3-3) The clause $\neg p_{i} \rightarrow \neg \square_{F} p_{i+1}$ for every $i$ with $1 \leq i<n$. Informally, these clauses (together with the clauses from C3-1 and C3-2) ensure that for every $i$ with $2 \leq i \leq n$, it holds that $p_{i}$ is false at the $i$-th world. Observe that the clauses from C3-1 to C3-3 already ensure that $\neg p_{i} \wedge \square_{F} p_{i}$ holds if and only if we are at the $i$-th world of the model for every $i$ with $1 \leq i \leq n$.

(C3-4) The clauses $\neg p_{n} \wedge \square_{F} p_{n} \rightarrow p_{n}^{\prime}$ and $\neg p_{n} \wedge \square_{F} p_{n} \leftarrow p_{n}^{\prime}=\neg p_{n} \wedge \square_{F} p_{n} \vee$ $\neg p_{n}^{\prime}=\left(\neg p_{n} \vee \neg p_{n}^{\prime}\right) \wedge\left(\square_{F} p_{n} \vee \neg p_{n}^{\prime}\right)$. Informally, these clauses (together with the clauses from C3-1 to C3-3) ensure that $p_{n}^{\prime}$ only holds in the $n$-th world of the model. Observe that all these clauses are HORN after removing the backdoor set variable $p_{n}^{\prime}$.

(C3-5) The clause $p_{n}^{\prime} \rightarrow \square_{P} p_{n}$. Informally, this clause (together with the clauses from C3-1 to C3-4) ensures that $p_{n}$ is only false in the $n$-th world of the model.

(C3-6) The clause $p_{i} \wedge \square_{P} p_{i} \rightarrow \square_{P} p_{i-1}$ for every $i$ with $2 \leq i \leq n$. Informally, these clauses (together with the clauses from C3-1 to C3-5) ensure that $p_{i}$ is true before the $i$-th world for every $i$ with $2 \leq i<n$. 


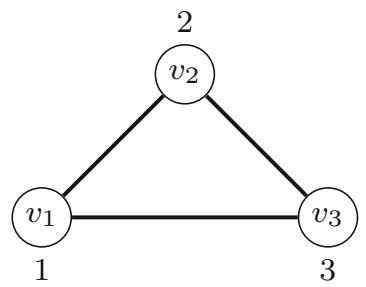

\begin{tabular}{ccccccccccccccccccc}
\hline \multicolumn{1}{c}{$s c_{1} c_{2} c_{3} p_{n}^{\prime}$} & $v_{1}^{1} v_{1}^{2} v_{1}^{3}$ & $v_{2}^{1}$ & $v_{2}^{2}$ & $v_{2}^{3}$ & $v_{3}^{1}$ & $v_{3}^{2}$ & $v_{3}^{3}$ & $p_{1}$ & $p_{2}$ & $p_{3}$ \\
\hline$<1$ & 0 & $*$ & $*$ & $*$ & 0 & 1 & 0 & 0 & 0 & 1 & 0 & 0 & 0 & 1 & 1 & 1 & 1 \\
1 & 1 & 1 & 0 & 0 & 0 & 1 & 0 & 0 & 0 & 1 & 0 & 0 & 0 & 1 & 0 & 1 & 1 \\
2 & 0 & 0 & 1 & 0 & 0 & 1 & 0 & 0 & 0 & 1 & 0 & 0 & 0 & 1 & 1 & 0 & 1 \\
3 & 0 & 0 & 0 & 1 & 1 & 1 & 0 & 0 & 0 & 1 & 0 & 0 & 0 & 1 & 1 & 1 & 0 \\
$>3$ & 0 & $*$ & $*$ & $*$ & 0 & 1 & 0 & 0 & 0 & 1 & 0 & 0 & 0 & 1 & 1 & 1 & 1 \\
\hline
\end{tabular}

Fig. 1 Left: A graph $G$ with vertices $v_{1}, v_{2}$, and $v_{3}$ together with a 3-coloring given by the numbers above and below respectively of every vertex. Right: A temporal interpretation $\mathfrak{M}$ that corresponds to the given 3 -coloring of $G$ and satisfies $\mathfrak{M} \models \phi$ given as a table. Each row of the table corresponds to a world (or a set of worlds) as indicated by the first column of the table. Each column represents the assignments of a variable as indicated in the first row. A “*” indicates that the assignment is not fixed, i.e., the assignment does not influence whether $\mathfrak{M} \models \phi$

Observe that all of the above clauses are HORN or become HORN after removing all variables from $B$. Note furthermore that all the above clauses ensure that $\square_{P} p_{i} \wedge \square_{F} p_{i}$ holds if and only if we are at the $i$-th world of the model for every $i$ with $1 \leq i \leq n$.

(C4) For every $i$ and $j$ with $1 \leq i \leq n$ and $1 \leq j \leq 3$ the clauses $\square_{F} p_{i} \wedge \square_{P} p_{i} \wedge$ $v_{i}^{j} \rightarrow c_{j}$ and $\square_{F} p_{i} \wedge \square_{P} p_{i} \wedge c_{j} \rightarrow v_{i}^{j}$. Informally, these clauses ensure that in the $i$-th world for every $1 \leq i \leq n$, the variables $c_{1}, c_{2}, c_{3}$ are a copy of the variables $v_{i}^{1}, v_{i}^{2}, v_{i}^{3}$. Observe that all of these clauses are HORN.

(C5) For every edge $e=\left\{v_{i}, v_{j}\right\} \in E(G)$ and every $c$ with $1 \leq c \leq 3$, the clause $\neg v_{i}^{c} \vee \neg v_{j}^{c}$. Informally, these clauses ensure that the 3-partition (of the vertices of $G$ ) given by the (global) values of the variables $v_{1}^{1}, v_{1}^{2}, v_{1}^{3}, \ldots, v_{n}^{1}, v_{n}^{2}, v_{n}^{3}$ is a valid 3-coloring for $G$. Observe that all of these clauses are HORN.

It follows from the definition of $\phi$ that $\phi[\theta] \in \mathrm{LTL}_{\text {HORN }}^{\square_{F}, \square_{P}}$ for every assignment $\theta$ of the variables in $B$. Consequently, $B$ is a strong HORN-backdoor of size four of $\phi$ as required. Moreover, since $\phi$ can be constructed in polynomial time, it only remains to show that $G$ has a 3-coloring if and only if $\phi$ is satisfiable.

Towards showing the forward direction assume that $G$ has a 3-coloring and let $f: V(G) \rightarrow\{1,2,3\}$ be such a 3 -coloring for $G$. We will show that $\phi$ is satisfiable by constructing a temporal interpretation $\mathfrak{M}$ such that $\mathfrak{M} \models \phi . \mathfrak{M}$ is defined as follows:

- For every $j$ with $1 \leq j \leq 3$, we set $\mathfrak{M}\left(c_{j}\right)=\left\{i \mid f\left(v_{i}\right)=j\right\}$.

- We set $\mathfrak{M}\left(p_{n}^{\prime}\right)=\{n\}$.

- For every $i$ and $c$ with $1 \leq i \leq n$ and $1 \leq c \leq 3$, we set $\mathfrak{M}\left(v_{i}^{c}\right)=\mathbb{Z}$ if $c=f\left(v_{i}\right)$ and otherwise we set $\mathfrak{M}\left(v_{i}^{c}\right)=\emptyset$.

- For every $i$ with $1 \leq i \leq n$, we set $\mathfrak{M}\left(p_{i}\right)=\mathbb{Z} \backslash\{i\}$.

An example for such a temporal interpretation resulting for a simple graph is illustrated in Figure 1. It is straightforward (but a little tedious) to verify that $\mathfrak{M} \models \phi$ by considering all the clauses of $\phi$.

Towards showing the reverse direction assume that $\phi$ is satisfiable and let $\mathfrak{M}$ be a temporal interpretation witnessing this. We will start by showing the following series of claims for $\mathfrak{M}$. 
(M1) For every $a \in \mathbb{Z}$ exactly one of $\mathfrak{M}, a \models c_{1}, \mathfrak{M}, a \models c_{2}$, and $\mathfrak{M}, a \models c_{3}$ holds.

(M2) For every $i, c, a$, and $a^{\prime}$ with $1 \leq i \leq n, 1 \leq c \leq 3$, and $a, a^{\prime} \in \mathbb{Z}$, it holds that $\mathfrak{M}, a \models v_{i}^{c}$ if and only if $\mathfrak{M}, a^{\prime} \models v_{i}^{c}$.

(M3) For every $i$ with $1 \leq i \leq n$ and every $a \in \mathbb{Z}$, it holds that $\mathfrak{M}, a \models p_{i}$ if and only if $a \neq i$.

(M4) For every $i$ and $j$ with $1 \leq i \leq n$ and $1 \leq j \leq 3$, it holds that $\mathfrak{M}, i \models c_{j}$ if and only if $\mathfrak{M}, i \models v_{i}^{j}$.

(M1) holds because of the clauses added by (C1). Towards showing (M2) consider the clauses added by $(\mathrm{C} 2)$ and assume for a contradiction that there are $i, c, a$, and $a^{\prime}$ as in the statement of (M2) such that w.l.o.g. $\mathfrak{M}, a \models v_{i}^{c}$ but $\mathfrak{M}, a^{\prime} \not \models v_{i}^{c}$. Then, $a \neq a^{\prime}$. If $a<a^{\prime}$, then we obtain a contradiction because of the clause $v_{i}^{c} \rightarrow \square_{F} v_{i}^{c}$ and if on the other hand $a^{\prime}<a$, we obtain a contradiction to the clause $v_{i}^{c} \rightarrow \square_{P} v_{i}^{c}$. This completes the proof of (M2). Considering the explanations for the clauses the proof of (M3) is now reasonably straightforward, however, for completeness we now provide a detailed proof. We will show (M3) with the help of the following series of claims.

(M3-1) For every $a \in \mathbb{Z}$ it holds that $\mathfrak{M}, a \models p_{1}$ if and only if $a \neq 1$ (here we assume that 1 is the starting world).

(M3-2) For every $i$ and $a$ with $1 \leq i \leq n, a \in \mathbb{Z}$, and $a>i$, it holds that $\mathfrak{M}, a \models p_{i}$. (M3-3) For every $i$ with $1 \leq i \leq n$, it holds that $\mathfrak{M}, i \not \models p_{i}$.

(M3-4) For every $a \in \mathbb{Z}$, it holds that $\mathfrak{M}, a \models p_{n}^{\prime}$ if and only if $a=n$.

(M3-5) For every $a \in \mathbb{Z}$, it holds that $\mathfrak{M}, a \not \models p_{n}$ if and only if $a=n$.

Because of the clause $s \rightarrow \neg p_{1}$ (added by C3-1) and the fact that $s \in \Psi$, we obtain that $\mathfrak{M}, 1 \not \models p_{1}$. Moreover, because of the clauses $s \rightarrow \square_{F} p_{1}$ and $s \rightarrow \square_{P} p_{1}$, we obtain that $\mathfrak{M}, a \models p_{1}$ for every $a \neq 1$. This completes the proof for (M3-1).

We show (M3-2) via induction on $i$. The claim clearly holds for $i=1$ because of (M3-1). Now assume that the claim holds for $p_{i-1}$ and we want to show it for $p_{i}$. Because of the induction hypothesis, we obtain that $\mathfrak{M}, i \models p_{i-1} \wedge \square_{F} p_{i-1}$. Moreover, because $\phi$ contains the clause $p_{i-1} \wedge \square_{F} p_{i-1} \rightarrow \square_{F} p_{i}$ (which was added by (C3-2)), we obtain that $\mathfrak{M}, i \models \square_{F} p_{i}$. This completes the proof of (M3-2).

We show (M3-3) via induction on $i$. The claim clearly holds for $i=1$ because of (M3-1). Now assume that the claim holds for $p_{i-1}$ and we want to show it for $p_{i}$. Because of the induction hypothesis, we obtain that $\mathfrak{M}$, $(i-1) \not \models p_{i-1}$. Furthermore, because of (M3-2), we know that $\mathfrak{M}, i \models \square_{F} p_{i}$. Since $\phi$ contains the clause $\neg p_{i-1} \rightarrow$ $\neg \square_{F} p_{i}$ (which was added by (C3-3)), we obtain $\mathfrak{M},(i-1) \models \neg \square F p_{i}$, which because $\mathfrak{M}, i \models \square_{F} p_{i}$ can only hold if $\mathfrak{M}, i \not \models p_{i}$. This completes the proof of (M3-3).

Towards showing (M3-4), first note that because of (M3-2) and (M3-3), we have that $\mathfrak{M}, a \models \neg p_{n} \wedge \square_{F} p_{n}$ if and only if $a=n$. Then, because of the clauses (added by C34) ensuring that $\neg p_{n} \wedge \square_{F} p_{n} \leftrightarrow p_{n}^{\prime}$, the same applies to $p_{n}^{\prime}$ (instead of $\neg p_{n} \wedge \square_{F} p_{n}$ ). This completes the proof of (M3-4).

It follows from (M3-2) and (M3-3) that (M3-5) holds for every $a \in \mathbb{Z}$ with $a \geq n$. Moreover, because of (M3-4), we have that $\mathfrak{M}, n \models p_{i}^{\prime}$. Because of the clause $p_{n}^{\prime} \rightarrow$ $\square_{P} p_{n}$ (which was added by (C3-5)), we obtain $\mathfrak{M}, a \models p_{n}$ for every $a<n$. This completes the proof of (M3-5). 
We are now ready to prove (M3). It follows from (M3-2) and (M3-3) that (M3) holds for every $i$ and $a$ with $a \geq i$. Furthermore, we obtain from (M3-5) that (M3) already holds if $i=n$. We complete the proof of (M3) via an induction on $i$ starting from $i=n$. Because of the induction hypothesis, we obtain that $\mathfrak{M}, i+1 \models p_{i+1} \wedge \square_{P} p_{i+1}$. Accordingly, because of the clause $p_{i+1} \wedge \square_{P} p_{i+1} \rightarrow \square_{P} p_{i}$ (added by (C3-6)), we obtain that $\mathfrak{M}, i+1 \models \square_{P} p_{i}$, which completes the proof of (M3).

Towards showing (M4) first note that it follows from (M3) that $\mathfrak{M}, i \models \square_{F} p_{i} \wedge$ $\square_{P} p_{i}$. Now suppose that there are $i$ and $j$ such that either $\mathfrak{M}, i \models c_{j}$ but $\mathfrak{M}, i \not v_{i}^{j}$ or $\mathfrak{M}, i \not c_{j}$ but $\mathfrak{M}, i \models v_{i}^{j}$. In the former case, consider the clause $\square_{F} p_{i} \wedge \square_{P} p_{i} \wedge c_{j} \rightarrow$ $v_{i}^{j}$ (which was added by (C4)). Since $\mathfrak{M}, i \models \square_{F} p_{i} \wedge \square_{P} p_{i}$, we obtain that $\mathfrak{M}, i \models v_{i}^{j}$; a contradiction. In the later case, consider the clause $\square_{F} p_{i} \wedge \square_{P} p_{i} \wedge v_{i}^{j} \rightarrow c_{j}$ (which was added by (C4)). Since $\mathfrak{M}, i \models \square_{F} p_{i} \wedge \square_{P} p_{i}$, we obtain that $\mathfrak{M}, i \models c_{j}$; again a contradiction. This completes the proof of the claims (M1)-(M4).

It follows from (M1) and (M4) that for every $i$ and $a$ with $1 \leq i \leq n$ and $a \in \mathbb{Z}$ there is exactly one $c$ with $1 \leq c \leq 3$, such that $\mathfrak{M}, a \models v_{i}^{c}$. Moreover, because of (M2) the choice of $c$ is independent of $a$. Accordingly, the coloring $f$ that assigns the unique color $c$ to every vertex $v_{i}$ such that $\mathfrak{M}, a \models v_{i}^{c}$ forms a partition of the vertex set of $G$. Also $f$ is a valid 3-coloring because for every $\left\{v_{i}, v_{j}\right\} \in E(G)$ it holds that $\mathfrak{M}, a \not \forall \neg v_{i}^{c} \vee \neg v_{j}^{c}$ for every $a \in \mathbb{Z}$ (using the clause added by C5) and hence $v_{i}$ and $v_{j}$ must be assigned distinct colors by $f$.

Corollary 11 Let $O \in\left\{\square_{\mathrm{F}}, \square_{\mathrm{P}}\right\}$ then $\mathrm{Eval}^{O}$ (KROM) is paraNP-complete (the NPcompleteness already holds for backdoor sets of size zero).

Proof Satisfiability of LTL ${ }_{\mathrm{KROM}}^{O}$ is NP-hard [2, Theorem 5].

\section{Conclusion and Discussion}

We lift the well-known concept of backdoor sets from propositional logic up to the clausal fragment of linear temporal logic LTL. From the investigated cases we obtain a comprehensive picture of the parameterized complexity for the problem of backdoor set evaluation. The evaluation parameterized by the size of the backdoor into KROM formulas becomes in all cases paraNP-complete and as a result is unlikely to be solvable in FPT whereas the case of backdoor evaluation into the fragment HORN behaves differently. While allowing only * makes the problem fixed-parameter tractable, allowing both, $\square_{\mathrm{F}}$ and $\square_{\mathrm{P}}$, makes it paraNP-complete. The last open case, i.e., the restriction to either $\square_{\mathrm{F}}$ or $\square_{\mathrm{P}}$ is open for further research and might yield an FPT result. We want to note here that all of our results still hold if LTL is evaluated over the natural numbers instead of the integers.

Satisfiability of LTL ${ }_{\mathrm{CNF}}^{\circledast}$ is NP-complete, for HORN/KROM it is in P/NL [2]. With the help of our backdoor notion, we achieved for a HORN-backdoor an FPT membership. However, for KROM this surprisingly was not possible (paraNP-c., Theorem 9). For the "full global" fragment only for HORN satisfiability is in P and for KROM it is NPcomplete [2]. Here in both cases, our notion of backdoors was not fruitful. This is, however, natural since applying the backdoor approach to a novel problem is never a 
simple nor straightforward task. We see our work as a first attempt to come up with such a notion for LTL, and, given the notorious difficulty of the LTL-satisfiability problem, we believe our tractability result for LTL formulas restricted to the always operator that are almost HORN is an encouraging result that justifies further investigation of this approach. As mentioned earlier, LTL restricted to the always operator, is already pretty interesting, since it allows one to express "Safety" properties of a system (e.g., $*(\neg x)$, where $x$ encodes something bad to happen). Also, see the work of Kupferman and Vardi on this topic [24]. Moreover, our intractability results for the remaining fragments of LTL indicate that a different notion of "closeness" is required to obtain tractability results for these fragments.

Acknowledgements The first and last author gratefully acknowledge the support by the German Research Foundation DFG for their grant ME 4279/1-1. The second and third author acknowledge support by the Austrian Science Fund (FWF, project P26696). We thank the anonymous referees for their valuable comments.

Open Access This article is distributed under the terms of the Creative Commons Attribution 4.0 International License (http://creativecommons.org/licenses/by/4.0/), which permits unrestricted use, distribution, and reproduction in any medium, provided you give appropriate credit to the original author(s) and the source, provide a link to the Creative Commons license, and indicate if changes were made.

\section{References}

1. Abu-Khzam, F.N.: A kernelization algorithm for d-hitting set. J. Comput. Syst. Sci. 76(7), 524-531 (2010)

2. Artale, A., Kontchakov, R., Ryzhikov, V., Zakharyaschev, M.: The complexity of clausal fragments of LTL. In: Proc. 19th LPAR, LNCS, vol. 8312 (2013)

3. Bauland, M., Schneider, T., Schnoor, H., Schnoor, I., Vollmer, H.: The complexity of generalized satisfiability for linear temporal logic. LMCS 5(1), 1-21 (2009)

4. Chen, C.C., Lin, I.P.: The computational complexity of satisfiability of temporal Horn formulas in propositional linear-time temporal logic. IPL 45(3), 131-136 (1993)

5. Chen, J., Chor, B., Fellows, M., Huang, X., Juedes, D., Kanji, I., Xia, G.: Tight lower bounds for certain parameterized NP-hard problems. Inf. Comput. 201(2), 216-231 (2005)

6. Chen, J., Kanj, I.A., Xia, G.: Improved upper bounds for vertex cover. Theor. Comput. Sci. 411(40-42), 3736-3756 (2010)

7. Clarke, E.M., Emerson, E.A.: Design and synthesis of synchronization skeletons using branching time temporal logic. Logic of Programs. LNCS, vol. 131, pp. 52-71. Springer, Berlin (1981)

8. Demri, S., Schnoebelen, P.: The complexity of propositional linear temporal logics in simple cases. Inf. Comput. 174(1), 84-103 (2002)

9. Dilkina, B.N., Gomes, C.P., Sabharwal, A.: Tradeoffs in the complexity of backdoor detection. In: Proc. 13th CP, Lecture Notes in Computer Science, vol. 4741, pp. 256-270. Springer (2007)

10. Dilkina, B.N., Gomes, C.P., Sabharwal, A.: Backdoors in the context of learning. In: Proc. 12th SAT, Lecture Notes in Computer Science, vol. 5584, pp. 73-79. Springer, Berlin (2009)

11. Dixon, C., Fisher, M., Konev, B.: Tractable temporal reasoning. In: Proc. of IJCAI, pp. 318-323 (2007)

12. Downey, R.G., Fellows, M.R.: Fundamentals of Parameterized Complexity. Springer, London (2013)

13. Dvorák, W., Ordyniak, S., Szeider, S.: Augmenting tractable fragments of abstract argumentation. Artif. Intell. 186, 157-173 (2012)

14. Emerson, E.A., Halpern, J.Y.: Decision procedures and expressiveness in the temporal logic of branching time. Journal of Computer and System Sciences 30(1), 1-24 (1985)

15. Fisher, M.: A normal form for temporal logic and its application in theorem-proving and execution. J. Logic Comput. 7, 429-456 (1997)

16. Fisher, M., Dixon, C., Peim, M.: Clausal temporal resolution. ACM Trans. Comput. Logic 2(1), 12-56 (2001) 
17. Gabbay, D.M., Hodkinsion, I., Reynolds, M.: Temporal Logic: Mathematical Foundations and Computational Aspects, vol. 1. Oxford University Press, Inc., New York (1994)

18. Gaspers, S., Szeider, S.: Backdoors to satisfaction. In: The Multivariate Algorithmic Revolution and Beyond - Essays Dedicated to Michael R. Fellows on the Occasion of His 60th Birthday, LNCS, vol. 7370, pp. 287-317. Springer, Berlin (2012)

19. Gaspers, S., Szeider, S.: Strong backdoors to bounded treewidth SAT. In: Proc. 54th FOCS, pp. 489498. IEEE Computer Society (2013)

20. Kottler, S., Kaufmann, M., Sinz, C.: A new bound for an NP-hard subclass of 3-SAT using backdoors. In: Proc. 11th SAT, Lecture Notes in Computer Science, pp. 161-167. Springer, Berlin (2008)

21. Kripke, S.: Semantical considerations on modal logic. Acta philosophica Fennica 16, 84-94 (1963)

22. Kronegger, M., Ordyniak, S., Pfandler, A.: Backdoors to planning. In: Proc. 28th AAAI, pp. 2300-2307. AAAI Press (2014)

23. Kronegger, M., Ordyniak, S., Pfandler, A.: Variable-deletion backdoors to planning. In: Proc. 29th AAAI, pp. 2300-2307. AAAI Press (2014)

24. Kupferman, O., Vardi, M.Y.: Model checking of safety properties. Formal Methods Syst. Des. 19(3), 291-314 (2001). https://doi.org/10.1023/A:1011254632723

25. LeBras, R., Bernstein, R., Gomes, C.P., Selman, B., van Dover, R.B.: Crowdsourcing backdoor identification for combinatorial optimization. In: Proc. 23rd IJCAI. AAAI (2013)

26. Lück, M., Meier, A.: LTL Fragments are hard for standard parameterizations. In: Proc. of TIME, pp. 59-68 (2015). https://doi.org/10.1109/TIME.2015.9

27. Markey, N.: Past is for free: on the complexity of verifying linear temporal properties with past. Acta Informatica 40(6-7), 431-458 (2004)

28. Meier, A., Ordyniak, S., Sridharan, R., Schindler, I.: Backdoors for linear temporal logic. In: J. Guo, D. Hermelin (eds.) 11th International Symposium on Parameterized and Exact Computation (IPEC 2016), vol. 63, pp. 23:1-23:17. Schloss Dagstuhl-Leibniz-Zentrum fuer Informatik (2017)

29. Ono, H., Nakamura, A.: On the size of refutation Kripke models for some linear modal and tense logics. Studia Logica 39, 325-333 (1980)

30. Ordyniak, S., Paulusma, D., Szeider, S.: Satisfiability of acyclic and almost acyclic CNF formulas. Theor. Comput. Sci. 481, 85-99 (2013)

31. Pfandler, A., Rümmele, S., Szeider, S.: Backdoors to abduction. In: Proc. 23rd IJCAI (2013)

32. Pnueli, A.: The temporal logic of programs. In: Proc. of FOCS, pp. 46-57. IEEE Comp. Soc. Press (1977)

33. Ruan, Y., Kautz, H.A., Horvitz, E.: The backdoor key: A path to understanding problem hardness. In: Proc. 19th IAAI, pp. 124-130. AAAI Press (2004)

34. Samer, M., Szeider, S.: Backdoor sets of quantified Boolean formulas. J. Autom. Reason. 42(1), 77-97 (2009)

35. Samer, M., Szeider, S.: Fixed-parameter tractability. In: Biere, A., Heule, M., van Maaren, H., Walsh, T. (eds.) Handbook of Satisfiability, chap 13, pp. 425-454. IOS Press, Amsterdam (2009)

36. Sistla, A., Clarke, E.: The complexity of propositional linear temporal logics. In: Proc. of STOC, pp. 159-168. ACM (1982)

37. Szeider, S.: On fixed-parameter tractable parameterizations of SAT. In: Proc. of SAT, pp. 188-202 (2003)

38. Williams, R., Gomes, C., Selman, B.: Backdoors to typical case complexity. In: Proc. 18th IJCAI, pp. 1173-1178. Morgan Kaufmann (2003)

Publisher's Note Springer Nature remains neutral with regard to jurisdictional claims in published maps and institutional affiliations. 